\title{
Centerline velocity decay of a circular jet in a counterflowing stream
}

\author{
C. H. C. Chan and K. M. Lam \\ Department of Civil and Structural Engineering, The University of Hong Kong, Pokfulam Road, \\ Hong Kong
}

(Received 10 February 1997; accepted 13 November 1997)

\begin{abstract}
We use an advection hypothesis to analyze the decay of centerline velocity of a circular jet issuing into a counterflowing stream. Working in the Lagrangian frame, we follow the locations and velocity gradients of jet fluid particles along the jet central axis while the particles are being advected backwards by the counterflow. The spatial velocity gradient along the jet centerline is thus obtained and subsequently integrated to describe the spatial decay of axial velocities. Laser-doppler velocity measurements are performed in the laboratory and the data are well predicted by our analytical expression of centerline velocity decay. Looking from another view, our treatment supports that the effect of an external axial flow stream on the jet flow field can be represented by a certain degree of stretching or contracting of the jet in the axial direction. (C) 1998 American Institute of Physics. [S1070-6631(98)01503-7]
\end{abstract}

\section{INTRODUCTION}

The investigation of jets in a stagnant ambient and in a moving current is of practical importance in many branches of engineering and science. The discharge of sewage and effluents into the sea or an estuary is one typical application in environmental engineering. The mean behavior of a circular jet in a stagnant ambient is quite well understood. Assuming self-similarity in the zone of established flow downstream of the potential core, the integral model shows that the centerline velocity exhibits a $x^{-1}$ dependence while the jet width varies linearly with $x$ (e.g., Rajaratnam, ${ }^{1}$ Liepmann and Laufer $^{2}$ ). For precision, the axial coordinate $x$ starts from a virtual origin which is upstream of the jet exit by a fraction of the jet exit diameter. The same analysis has been applied to a circular jet in a coflowing stream and the asymptotic solutions show that in a weak coflowing stream, the jet exhibits the same $x$ dependence as the simple jet while in a strong coflow, the centerline velocity and the jet width tend to follow a $x^{-2 / 3}$ and $x^{1 / 3}$ dependence, respectively. ${ }^{1}$ Available experimental data of jet in a coflow were reviewed by Woods. ${ }^{3}$ Extensive experimental studies of a jet in a cross flow were also reported by many authors, e.g., Andrepoulos and Rodi. ${ }^{4}$ However, there has not been an analytical method to describe the centerline velocity of a jet in a counterflowing stream, that is, a circular jet which is issuing into the same ambient fluid having a uniform flow in the opposing direction as the jet.

Experimental observations show that in a counterflow, the jet penetrates an axial distance of $l_{p}$ and is then deflected backwards. ${ }^{5}$ Along the penetrating distance, the jet centerline velocity is expected to drop more rapidly than in a nonflowing ambient and become stagnant at $l_{p}$. This penetration distance depends on the jet-to-current velocity ratio and a linear dependence was suggested by Sekunkov ${ }^{6}$ and Rajaratnam $^{1}$ from dimensional considerations. Recently, Yoda and Fiedler $^{7}$ have used laser-induced fluorescence (LIF) to study the structure and concentration field of the counterflowing jet. They also found that the penetration distance increases with the velocity ratio.

In this paper, we attempt to analyze the centerline velocity decay in the counterflowing jet with a Lagrangian treatment. By relative motion, a jet in counterflow is physically equivalent to a towing jet in stagnant ambient. In this way, we first solve for the shorter potential core length of the counterflowing jet. We then consider the kinematics of a fluid element issued from the end of the potential core into the zone of established flow where fluid particles traveling along the jet centerline still tend to have their velocities dropping with $x^{-1}$. However, the velocity decay is modified by the presence of the counterflow. The counterflow is proposed to exert an advection effect on the fluid particles, thus compressing distances between successive particles. Using this advection hypothesis, we can define the velocities and axial locations of two successive fluid particles which the jet issues along its central axis in the zone of established flow. The spatial derivative of particle velocity is thus obtained and is integrated to give the centerline velocity decay.

\section{FLOW ESTABLISHMENT IN THE POTENTIAL CORE}

A jet flow field is conveniently divided into the potential core region and the zone of established flow. In a stagnant ambient, many studies suggested that the length of the potential core, $l$, is independent of jet exit velocity and has a value of $6.2 \mathrm{D}^{8}{ }^{8}$ For a jet with exit velocity $U_{j}$ in a counterflow $U_{0}$, we can observe the flow with a frame of reference moving with the counterflow. Now we have the jet issuing at an exit velocity $\left(U_{j}+U_{0}\right)$ into a stagnant ambient and the nozzle is moving in the jet exit direction with $U_{0}$. Initial instabilities in the shear layer and the subsequent vortex rollup lead to the formation of vortex rings. The rings grow in the shear layer by entrainment and pairing as they travel downstream until their size becomes comparable to the radius of the jet. Then they break down at the end of the potential core as reported by Liepmann et al. ${ }^{9}$ In the shear 
layer, the vortex rings can be assumed to travel with a velocity $U_{v}=\left(U_{j}+U_{0}\right) / 2$ (see Fig. 1). A vortex ring then takes a time $t=l / U_{v}$ to reach the end of the potential core from the nozzle. In this time interval, the nozzle has advanced forward by a displacement $U_{0} t$, which is equal to $l U_{0} / U_{v}$. Thus, the potential core length $l_{c}$ as it appeared to an observer moving with the nozzle is reduced to

$$
l_{c}=l-l U_{0} / U_{v}=l\left(U_{j}-U_{0}\right) /\left(U_{j}+U_{0}\right) .
$$

The shortening of the potential core of the jet in the presence of a counterflow can be argued to be a consequence of the interaction of forward jet momentum with the negative momentum of the counterflow, an issue which will be discussed next.

For a round jet in a stagnant ambient, length scale analysis and experimental data have established that downstream of the potential core, the centerline velocity $U_{c}$ in the jet decays with an $x^{-1}$ dependence. ${ }^{8}$ The following expression of $U_{c}$, in terms of the jet momentum flux $M$, applies in the zone of the established flow:

$$
U_{c}=B M^{1 / 2} / x, \quad x>l .
$$

The constant $B$ has been found to have a value 7.0. In a simple jet, momentum flux is conserved and its value is equal to $\pi / 4 D^{2} U_{j}^{2}$. Since the centerline jet starts to drop from $U_{j}$ at the end of the potential core, we choose the following expression for $U_{c}$ in terms of the potential core length $l$ by substituting $M=\pi / 4 D^{2} U_{j}^{2}$ into Eq. (2):

$$
U_{c}=l U_{j} / x, \quad x>l,
$$

where $l=6.2 \mathrm{D}$ is the potential core length in stagnant ambient. Equations (2) and (3) are equivalent, with $B=7.0$ and $l=6.2 \mathrm{D}$.

In the presence of a counterflow, however, we cannot use Eq. (3) with the shorter potential core length $l_{c}$ in Eq. (1). The effect of the counterflow on the momentum flux needs to be looked at and Eq. (2) modified accordingly. Simply stated, the jet in a counterflow entrains fluids which carries negative momentum and as a result, the forward momentum flux $M_{*}$, at the end of the potential core is smaller than $M$.

In the zone of flow establishment, we model the growth of jet shear layer by simple toroidal vortex rings which grow linearly in size (Fig. 1). Forced vortex flow up to the vortex radius is assumed. For a round jet in a stagnant ambient, the peripheral velocity induced by the vortex is $\frac{1}{2} U_{j}$. At the end of the potential core, the vortex ring has grown to a size which extends the entire jet half-width there. Taking the jet half-width as $k D$ there, the angular velocity of the vortex at the end of the potential core is given by the peripheral velocity divided by the vortex radius,

$$
\omega=-\frac{1}{2} U_{j} / \frac{1}{2} k D=-U_{j} / k D,
$$

where the negative sign indicates counterclockwise rotation.

The vortex ring is located with its center located at $k D / 2$ from the jet center line, so that the circulation velocity varies as with the radial coordinate as

$$
U(r)=-U_{j}(r-k D / 2) / k D, \quad r \leqslant k D
$$

(a)

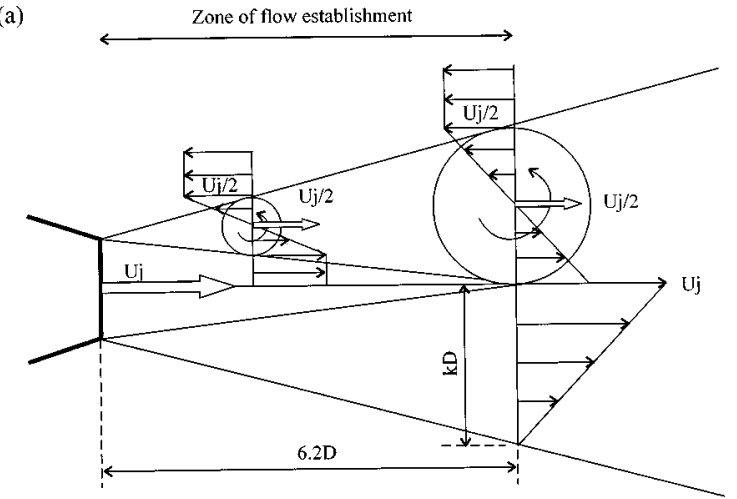

(b)

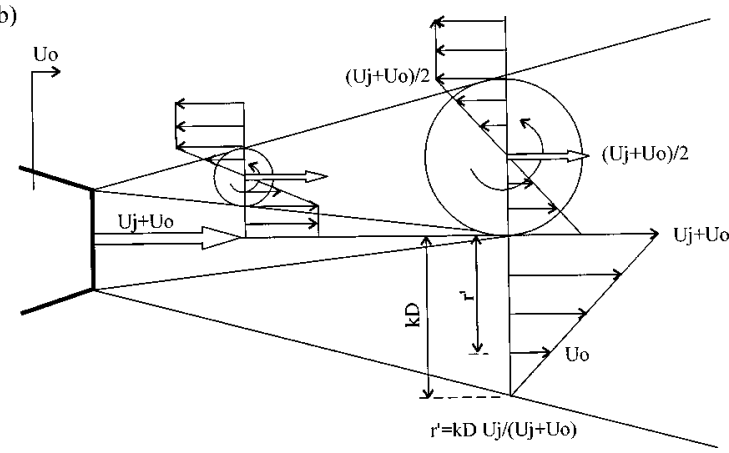

FIG. 1. Toroidal vortex rings modeled in the jet shear layer in the region of flow establishment: (a) in stagnant ambient; (b) in a counterflow.

In the shear layer, the vortex has been traveling at a speed $\frac{1}{2} U_{j}$ so that at the jet center line, the forward velocity is $U_{j}$. Adding a uniform velocity $\frac{1}{2} U_{j}$ to the circular velocity field, the radial velocity profile of the jet at the end of potential core is

$$
U(r)=(1-r / k D) U_{j}, \quad r \leqslant k D .
$$

The momentum flux at the end of the potential core is obtained as

$$
M=\int_{0}^{k D}\left[U_{j}(1-r / k D)\right]^{2} 2 \pi r d r=(\pi / 6) U_{j}^{2}(k D)^{2}
$$

Now, we consider a counterflowing jet by taking a jet with exit velocity $\left(U_{j}+U_{0}\right)$ in stagnant ambient but with the nozzle moving $U_{0}$ forward. Thus, the forward momentum flux at the end of the potential core, relative to the moving nozzle, is

$$
\left.M_{*}=\int_{0}^{r^{\prime}}\left\{\left[U_{j}+U_{0}\right)(1-r / k D)-U_{0}\right]\right\}^{2} 2 \pi r d r,
$$

where $r^{\prime}=k D\left(U_{j} /\left(U_{j}+U_{0}\right)\right)$ is the radial position where the velocity is $U_{0}$ in Fig. 1(b). Integrating

$$
M_{*}=(\pi / 6) U_{j}^{2}\left[k D U_{j} /\left(U_{j}+U_{0}\right)\right]^{2},
$$

which is smaller than the forward momentum flux in Eq. (5) for a simple jet,

$$
M_{*}=\left[U_{j} /\left(U_{j}+U_{0}\right)\right]^{2} M .
$$

Adopting a similar form as Eq. (2) based on length scale analysis, the centerline velocity decays in the zone of established flow as 


$$
U_{c}=B M_{*}^{1 / 2} / x=B\left[U_{j} /\left(U_{j}+U_{0}\right)\right] M^{1 / 2} / x .
$$

The expression can be simplified to a form similar to Eq. (3):

$$
U_{c}=l_{M} U_{j} / x .
$$

This characteristic length $l_{M}$ is defined by momentum considerations. In order for Eq. (9) to be equivalent the to Eq. (8), and noting that $B M^{1 / 2}=l U_{j}$ from Eq. (3), the characteristics length $l_{M}$ is related to the simple jet potential core length by

$$
l_{M}=l U_{j} /\left(U_{j}+U_{0}\right) .
$$

It is noted that this length $l_{M}$ from momentum consideration is longer than the counterflowing jet potential core length $l_{c}$ as derived in Eq. (1). This suggests that for $x^{-1}$ decay, the axial coordinate $x$ should start from an origin further shifted by a position $d_{v}$ given by

$$
d_{v}=l_{M}-l_{c}=U_{0} /\left(U_{j}+U_{0}\right) l .
$$

The centerline velocity decay expression in the zone of the established flow of a jet in counterflow, Eq. (9), thus becomes

$$
U_{c}=l_{M} U_{j} / x^{*} \text { for } x>l_{c},
$$

where

$$
x^{*}=x+d_{v} .
$$

In order to simplify the symbolic notation, the symbol $x$ is still adopted instead of $x^{*}$ in subsequent equations.

\section{LAGRANGIAN FORMULATION IN THE ZONE OF ESTABLISHED FLOW}

In Sec. II, we established equations to account for the effect of counterflow on the establishment of jet flow up to the end of the potential core and the initial centerline velocity decay beyond the potential core. In counterflow, we model a fluid particle issued along the jet centerline that will first travel with a velocity $U_{j}$ but its velocity will decay as a consequence of two effects. The first effect is due to normal jet spreading so that the velocity tends to decay with $x^{-1}$ as if in a stagnant ambient. The presence of the counterflow exerts another effect which tends to advect the fluid particle backwards with $U_{0}$ so that the fluid particle will be pushed backwards with a finite displacement. However, as it travels downstream, it cannot be advected further backwards due to the fact that successive fluid particles are being continuously issued into the zone of established flow; momentum exchange will take place at the same time, resulting in a drop in the particle velocity. With this hypothesis, we shall follow the Lagrangian motion of the successive particles along the jet centerline and work out the expression of the velocity decay. It should be noted that in real situations, there exists a transition region between the potential core and the zone of established flow where the jet centerline velocity changes smoothly from the jet exit velocity to the $x^{-1}$ decay in an asymptotic manner.
Consider a fluid particle A, leaving the end of potential core at time $t=0$, and traveling afterwards with a velocity $U_{c}$ as given by Eq. (10). Accordingly, the distance which it has traveled over any interval $d t$ is given by

$$
d x=U_{c} d t .
$$

Now let us consider another fluid particle B which follows particle A and leaves the end of potential core at time $t$ $=d t$ so that it is now at a distance $d x$ from A. From the velocity-space equation of Eq. (10) of a jet in a stagnant ambient, the spatial gradient of jet centerline velocity is obtained by differentiating Eq. (10) as

$$
d U_{c} / d x=-U_{j} l_{M} x^{-2} .
$$

Thus, the velocity of particle A differs from that of B by

$$
d U=-U_{j} l_{M} x^{-2} d x .
$$

In the time interval $d t$, A has traveled a distance $d x$ as given by Eq. (12), thus the spatial separation between particles $A$ and $\mathrm{B}$ is

$$
x_{\mathrm{A}}-x_{\mathrm{B}}=U_{j} l_{M} / x d t .
$$

Combining the above two equations (with $x_{\mathrm{A}}-x_{\mathrm{B}}=d x$ ), we have

$$
d U=-\left(U_{j} l_{M}\right)^{2} x^{-3} d t,
$$

where $x$ is taken as $x_{\mathrm{B}}$. However, due to the advection effect suggested above, particle $\mathrm{A}$ is advected a distance $U_{0} d t$ backward by the counterflow. Particle B, on the other hand, is just at the end of the potential core and has not entered the zone of established flow, thus it is not yet being affected by the advection. Therefore, as shown in Fig. 2, their spatial separation is reduced to

$$
d x=x_{\mathrm{A}}-x_{\mathrm{B}}=\left(U_{j} l_{M} / x_{\mathrm{B}}-U_{0}\right) d t .
$$

Combining Eqs. (15) and (16), we can obtain an expression describing the spatial variation of particle velocity as

$$
d U / d x=F(x)=-U_{j} l_{M} / x^{2}-U_{0} / x+U_{0}^{2} /\left(U_{0} x-U_{j} l_{M}\right) .
$$

Equation (17) can only be applied up to $x_{\mathrm{B}}$ at this point. [Note that the velocity gradient of $\mathrm{AB}$ is chosen to be represented by $\mathrm{B}$, the rear edge of the element $\mathrm{AB}$, i.e., $F\left(x_{\mathrm{B}}\right)$ ], but we will now show that the function $F$ is valid up to the penetration distance.

We now treat $A B$ as a single fluid element and trace it downstream to a new position after some time $d \tau$ such that B will reach the previous location of A. Mathematically,

$$
x_{\mathrm{B}}^{\prime}=x_{\mathrm{A}},
$$

where $x_{\mathrm{B}}^{\prime}$ is the position of $\mathrm{B}$ at time $\tau$. According to the hypothesis we suggest, the element $\mathrm{AB}$ decays under the $1 / x$ law (normal jet decay in stagnant ambient) and undergoes an advection. However, it cannot be advected further backwards due to the fact that new elements behind $\mathrm{AB}$ are discharged continuously. This prevents element AB from moving backwards. As a result of momentum exchange with neighboring fluid elements, the velocity of the $\mathrm{AB}$ drops, say by $\delta \nu$, so as to achieve continuity of velocity. Thus A will follow the 


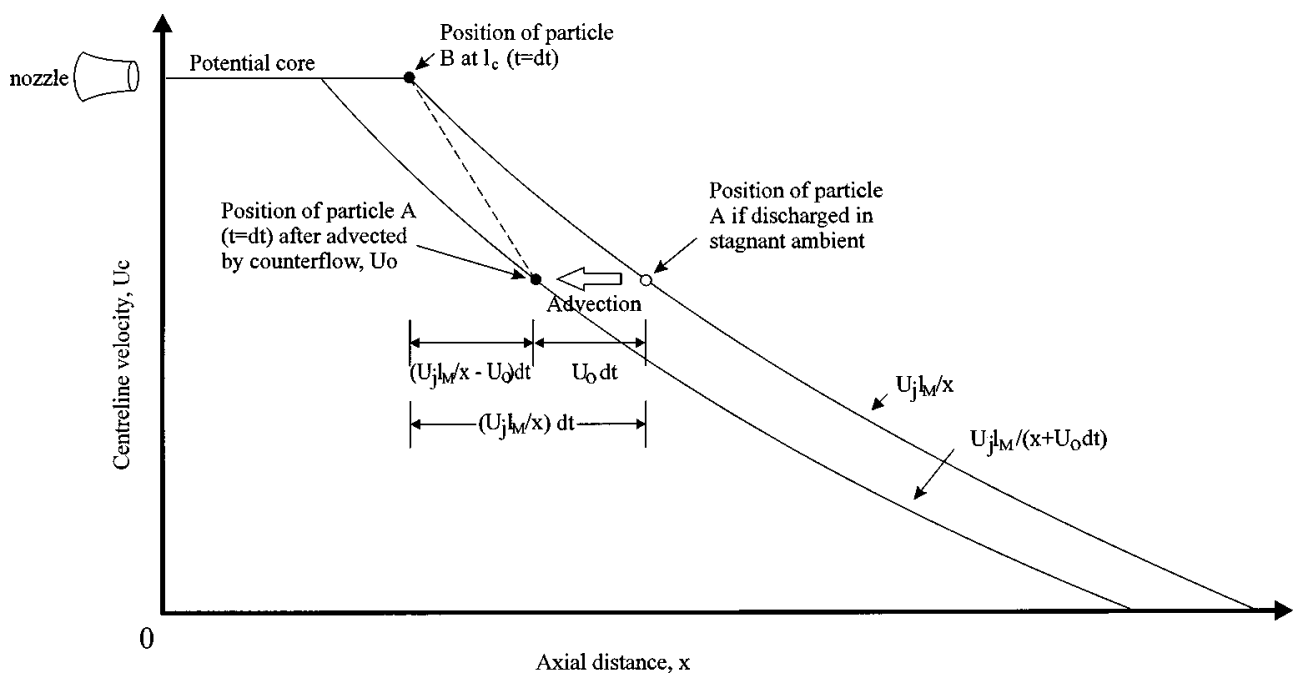

FIG. 2. Backward advection of a fluid particle issued from the end of potential core.

velocity-space curve below as shown in Fig. 3 (note that the term $U_{0} d t$ is due to A advected backwards, so the velocity space curve is shifted back by the value of $U_{0} d t$ ),

$$
U_{\mathrm{A}}=U_{j} l_{M} /\left(x_{\mathrm{A}}+U_{0} d t\right)-\delta \nu=K /\left(x_{\mathrm{A}}+U_{0} d t\right)-\delta \nu,
$$

where $K$ is used for $U_{j} l_{M}$ here and in the coming equations for simplicity. Similarly, B will travel with a velocity which varies with distance as

$$
U_{\mathrm{B}}=K / x_{\mathrm{B}}-\delta \nu .
$$

[Detailed arguments of particles A and B following Eqs. (19) and (20), respectively, are given in the Appendix.]

At the new time $\tau$, which is $d \tau$ from the previous time $d t$, the new position $x_{\mathrm{A}}^{\prime}, x_{\mathrm{B}}^{\prime}$ will be

$$
\begin{aligned}
& x_{\mathrm{A}}^{\prime}=x_{\mathrm{A}}+U_{\mathrm{A}} d \tau=x_{\mathrm{A}}+\left[K /\left(x_{\mathrm{A}}+U_{0} d t\right)-\delta \nu\right] d \tau, \\
& x_{\mathrm{B}}^{\prime}=x_{\mathrm{B}}+U_{\mathrm{B}} d \tau=x_{\mathrm{B}}+\left(K / x_{\mathrm{B}}-\delta \nu\right) d \tau .
\end{aligned}
$$

The new separation between particles A and B becomes

$$
x_{\mathrm{A}}^{\prime}-x_{\mathrm{B}}^{\prime}=x_{\mathrm{A}}-x_{\mathrm{B}}+\left[K /\left(x_{\mathrm{A}}+U_{0} d t\right)-K / x_{\mathrm{B}}\right] d \tau .
$$

Using (16), this reduces to

$$
\begin{aligned}
x_{\mathrm{A}}^{\prime}-x_{\mathrm{B}}^{\prime} & =\left(K / x_{\mathrm{B}}-U_{0}\right) d t+\left[K /\left(x_{\mathrm{A}}+U_{0} d t\right)-K / x_{\mathrm{B}}\right] d \tau \\
& =\left[K /\left(x_{\mathrm{A}}+U_{0} d t\right)-U_{0}\right] d \tau .
\end{aligned}
$$

As $x_{\mathrm{A}} \gg U_{0} d t$,

$$
x_{\mathrm{A}}^{\prime}-x_{\mathrm{B}}^{\prime}=\left(K / x_{\mathrm{A}}-U_{0}\right) d \tau .
$$

From Eq. (18),

$$
x_{\mathrm{A}}^{\prime}-x_{\mathrm{B}}^{\prime}=\left(K / x_{\mathrm{B}}^{\prime}-U_{0}\right) d \tau \text {. }
$$

The new difference in velocities of particles A and B is

$$
d U=\left[K /\left(x_{\mathrm{A}}^{\prime}+U_{0} d t\right)-\delta \nu\right]-\left(K / x_{\mathrm{B}}^{\prime}-\delta \nu\right) .
$$

From Eq. (23),

$$
x_{\mathrm{A}}^{\prime}=x_{\mathrm{B}}^{\prime}+\left(K / x_{\mathrm{B}}^{\prime}-U_{0}\right) d \tau \text {. }
$$

Thus,

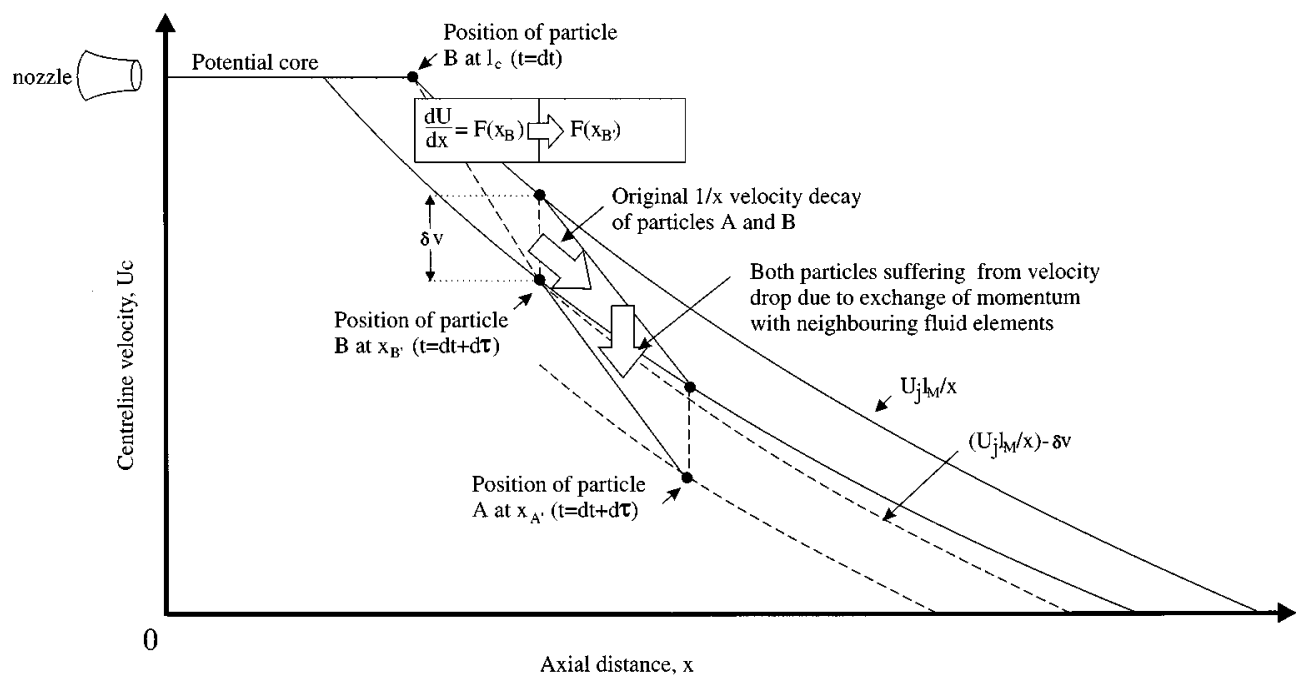

FIG. 3. Interaction of the fluid element at the next time step after being issued from the end of potential core. 


$$
d U=K /\left[x_{\mathrm{B}}^{\prime}+\left(K / x_{\mathrm{B}}^{\prime}-U_{0}\right) d \tau+U_{0} d t\right]-K / x_{\mathrm{B}}^{\prime} .
$$

Assuming $d \tau \approx d t$, we have

$$
d U=-\left(K / x_{\mathrm{B}}^{\prime}\right) d \tau /\left[x_{\mathrm{B}}^{\prime}+\left(K / x_{\mathrm{B}}^{\prime}\right) d \tau\right]\left(x_{\mathrm{B}}^{\prime}\right) .
$$

As $x_{\mathrm{B}}^{\prime} \gg\left(K / x_{\mathrm{B}}^{\prime}\right) d \tau$,

$$
d U=-d \tau / x_{\mathrm{B}}^{\prime 3} .
$$

Now the velocity gradient is obtained from Eqs. (23) and (24) as

$d U / d x=F\left(x_{\mathrm{B}}^{\prime}\right)=-U_{j} l_{M} / x_{\mathrm{B}}^{\prime 2}-U_{0} / x_{\mathrm{B}}^{\prime}+U_{0}^{2} /\left(U_{0} x_{\mathrm{B}}^{\prime}-U_{j} l_{M}\right)$.

Thus, the expression of $F(x)$ for the velocity gradient has been shown to be valid at the new time $d \tau$ when the fluid element $\mathrm{AB}$ is at $x_{\mathrm{B}}^{\prime}$. A similar argument can be applied to a subsequent time step to verify that the expression $F(x)$ is valid at any position along the jet centerline. The spatial variation of centerline velocity can be found by integration:

$$
\begin{aligned}
U_{c}= & \int(d U / d x) d x \\
& =\int\left[-U_{j} l_{M} / x^{2}-U_{0} / x+U_{0}^{2} /\left(U_{0} x-U_{j} l_{M}\right)\right] d x \\
& =U_{j} l_{M} / x+U_{0} \log \left(U_{j} l_{M} / x-U_{0}\right)+C .
\end{aligned}
$$

The constant $C$ can be found with the initial condition $U_{c}$ $=U_{j}$ at $x=l_{M}$ as

$$
C=-U_{0} \log \left(U_{j}-U_{0}\right) \text {. }
$$

Thus the centerline velocity decay is obtained in terms of $U_{j}, U_{0}$, and the actual $x^{*}$ as given by Eq. (11):

$$
\begin{aligned}
U_{c}= & U_{j} l_{M} / x^{*}+U_{0} \log \left(U_{j} l_{M} / x^{*}-U_{0}\right) \\
& -U_{0} \log \left(U_{j}-U_{0}\right), \quad x^{*}>l_{M} .
\end{aligned}
$$

The penetration distance $l_{p}$ of the counterflowing jet is defined as the point where the centerline velocity decays to zero. The value of $l_{p}$ can be found from Eq. (27). Figure 4(a) shows the centerline velocity decay curves predicted by this equation for a number of velocity ratios $U_{j} / U_{0}$. For the lower three velocity ratios, experimental data of penetration distances are available from Yoda and Fiedler, ${ }^{7}$ while experiments on counterflowing jets at the other five velocity ratios $U_{j} / U_{0}=3.09,5.03,7.5,10$, and 15 are carried out in the present study.

In order to examine whether there is a simple power law of decay for the axial mean velocities, the predicted centerline velocity decay curve are replotted in the $\log -\log$ form in Fig. 4(b). At all velocity ratios, the velocities do not decay with a constant exponent. Toward the penetration length, the exponent of decay, if a power law is assumed, keeps on increasing. Unlike a coflowing jet, in which the velocity decay asymptotes to an $x^{-2 / 3}$ power law, ${ }^{2}$ the data here do not follow any particular power decay law. The rate of decay depends on the jet-to-current ratio; the smaller the ratio, the more rapid is the decay. However, the data just downstream of the potential core tend to follow an $x^{-1}$ law. This obser-
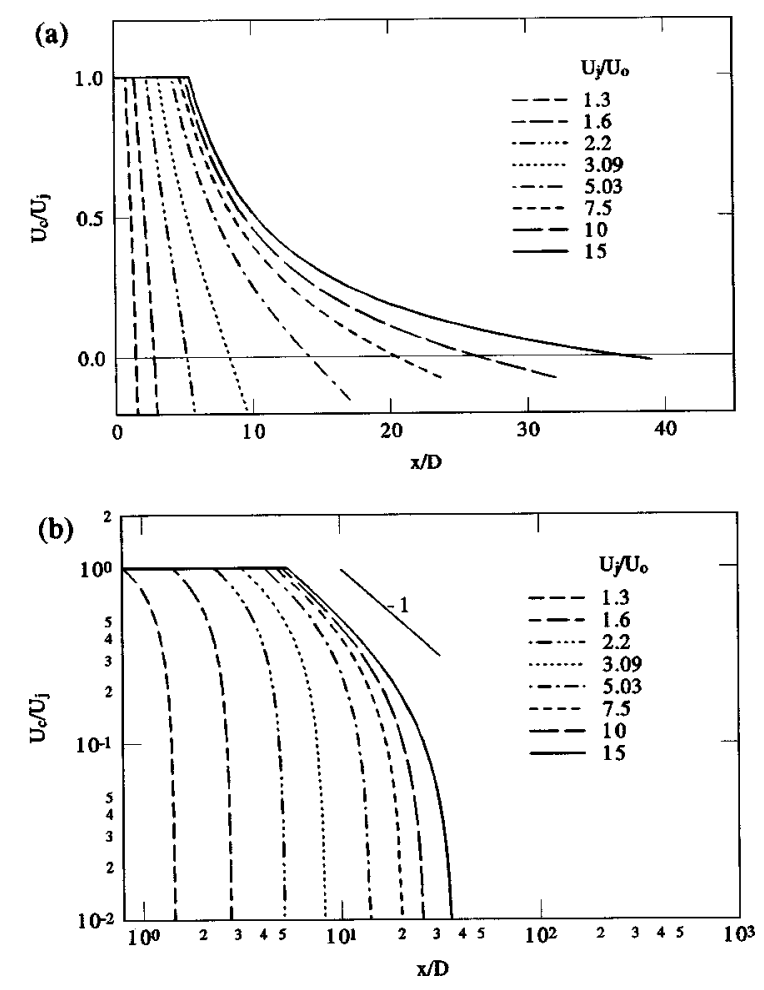

FIG. 4. Prediction of centerline velocity decay of the jet in a counterflow.

vation is more distinct at higher jet-to-current ratios where the effect of the counterflowing current is weaker.

In Sec. IV, we shall describe our experimental results at the higher velocity ratios.

\section{EXPERIMENT}

We carried out some experiments in a $10 \mathrm{~m} \times 0.45 \mathrm{~m} \times 0.3 \mathrm{~m}$ wide laboratory flume. A counterflowing jet was formed by issuing water from a circular nozzle against the main flow stream of the flume. The horizontal nozzle had an exit diameter of $D=10 \mathrm{~mm}$ and was fed from a constant head tank. The nozzle was located horizontally at the center of the flume and at the middepth of the main flow. The jet velocities $U_{j}$ ranged from 3 to 15 times the magnitude of the counterflowing current. The jet Reynolds number thus ranged from 3000 to 15000 . The ambient flow velocity in the flume was kept constant at a fixed value of $U_{0}$ $=10 \mathrm{~cm} / \mathrm{s}$, while the jet exit velocity $U_{j}$ was adjusted to give a range of jet-to-current velocity ratios $U_{j} / U_{0}$. To investigate the effect of the finite width between the walls of the flume on the spreading of the jet at higher velocity ratios, experiments were repeated at $U_{j} / U_{0}=7.5$ and 15 with a smaller jet nozzle of diameter $5.3 \mathrm{~mm}$.

Velocities along the jet central axis were measured with a DANTEC two-color fiber-optic laser-Doppler anemometer (LDA). Measurements were performed in backscatter mode with a $3 \mathrm{~W}$ argon-ion laser and two counterprocessors (DANTEC 55L90a) with frequency shift. The flow is seeded using pollycrystalline powder which is neutrally buoyant with a nominal diameter $10 \mu \mathrm{m}$.

Figure 5 shows the measurement data of axial mean velocities $U$ at the same five velocity ratios as in Fig. 4. The 


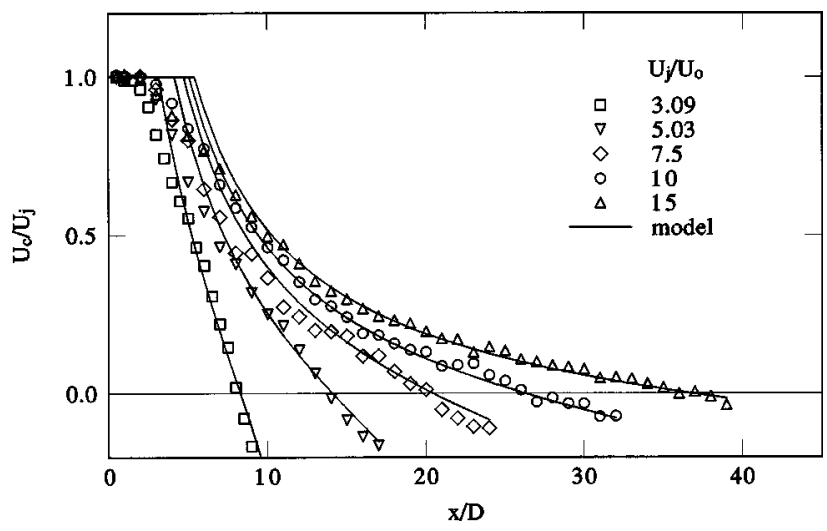

FIG. 5. Comparison of the predicted centerline velocity decay with experimental results.

velocity decay curves as predicted by Eq. (27) are also plotted in Fig. 5. We can observe that our analytical model predicts the velocity decay satisfactorily at all velocity ratios except at the start of the zone of established flow. These discrepancies are expected because in this transition region, the flow has not yet fully reached self-similarity. Beyond the penetration distance, LDA measurements have been made at locations as far downstream as where $U_{c}$ becomes $0.8 U_{0}$ in the counterflow direction. The model prediction still agrees well with the data there. In our model, we have assumed that the $1 / x$ decay of the centerline velocity extends to infinite distance but it must be recognized that when the centerline velocity approaches the counterflow velocity, the effect of the counterflow becomes dominant over the jet flow and the analysis will not be valid. Thus Eq. (27) should only be applied after the potential core length and before the position where the centerline velocity becomes the ambient flow velocity. The lateral mean velocities $V$ were also measured but are not shown because they all have very small values near zero, which serve to show that the LDA measurements had been performed on the jet central axis. A number of velocity profiles across traverse sections of the jet have also been measured and the results, not shown here, support the position that the jet central axis chosen in the laboratory coordinate falls very close to the actual jet central axis.

Previously, we have carried out laser-induced fluorescence (LIF) measurements on the penetration of the circular jet into the counterflow. The details have been reported in Lam and Chan. ${ }^{5}$ Essentially, we marked the jet with a fluorescent dye Rhodamine-G and illuminated a longitudinal section of the jet with a laser sheet. Then we performed ensemble averaging on the fluorescence levels over a large number of flow images to obtain the time-averaged pattern of jet penetration and spreading. An example of an averaged LIF picture is shown in Fig. 6. Penetration distances at different velocity ratios have been measured from these LIF pictures and are reproduced here in Fig. 7. In the present investigation, we repeated LIF measurements at $U_{j} / U_{0}$ $=7.5$ and 15 using a smaller nozzle with $D=5.3 \mathrm{~mm}$, with an aim to minimize the effect of the finite width of the laboratory flume. As shown in Fig. 7, these penetration distance data are in line with our previous main set of data. In the

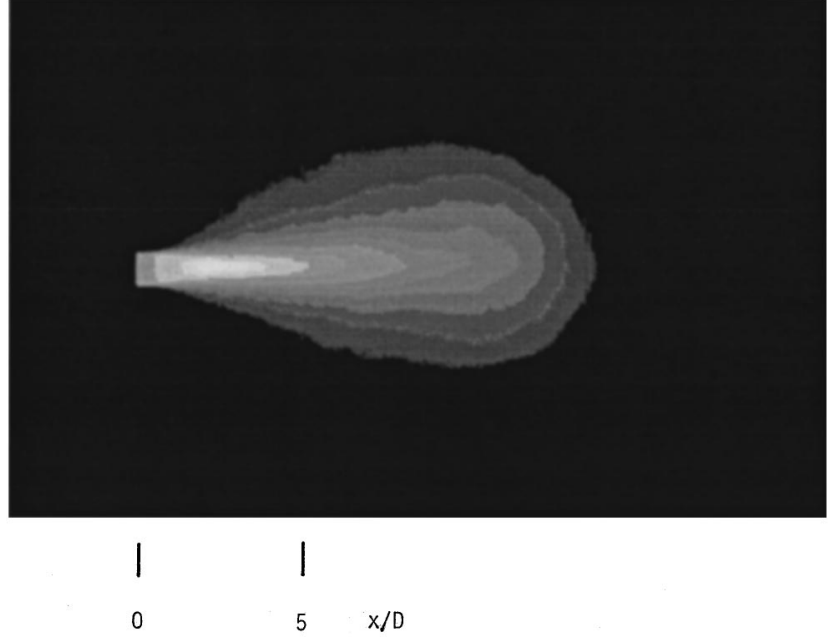

FIG. 6. Ensemble averaged LIF picture. $U_{j} / U_{0}=5$.

present model, the penetration distance can be obtained from Eq. (27) as the distance at which the centerline velocity decays to zero. The solid line in Fig. 7 shows the variation of $l_{p} / D$ with velocity ratios, as predicted by our model. Also shown is the empirical linear relationship $l_{p} / D=2.4 U_{j} / U_{0}$ or $2.7 U_{j} / U_{0}$, which was suggested by early studies in Rajaratnam. ${ }^{1}$ It is evident that the LIF data lie more closely on our prediction.

Experimental data of penetration distance at velocity ratios as low as 1.3 up to 10 are available from Yoda and Fiedler. $^{7}$ Their data are included in Fig. 7. For the higher ratios, the data agree with our model as well as with the linear relationship $l_{p} / D=2.7 U_{j} / U_{0}$. For $U_{j} / U_{0}$ below 2, it is obvious that the experimental data agree better with our model. There has been another investigation by Morgan et al. ${ }^{10}$ on the penetration of a turbulent jet into a counterflowing turbulent pipe flow which covered very high values of velocity ratios. They suggested that based on the jet momentum, there exist two flow regimes in which the penetration distances vary with velocity ratios in a different manner. In the high jet momentum regime, the jet is confined by the

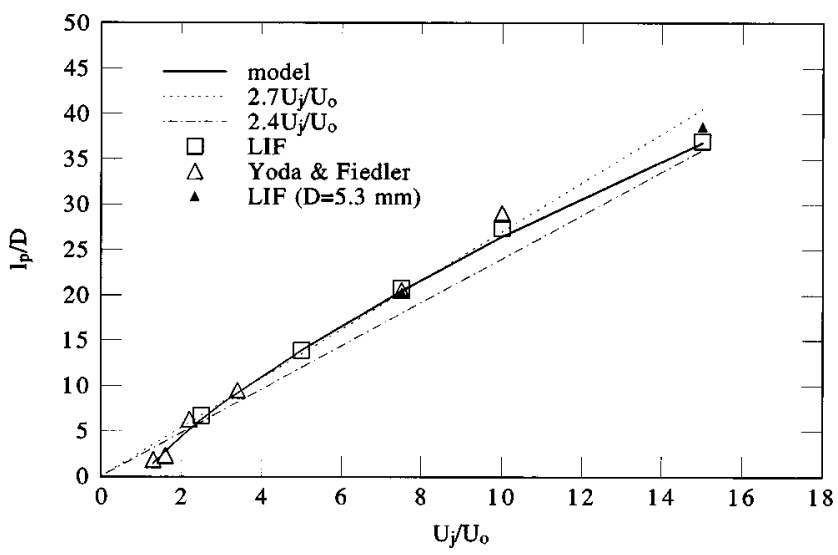

FIG. 7. Dependence of penetration distance on jet-to-current velocity ratio:- present prediction; $\square$, LIF results from Lam and Chan (Ref. 5); $\triangle$, LIF results from Yoda and Fiedler (Ref. 7); $\boldsymbol{\Delta}$, LIF results with nozzle diameter $5.3 \mathrm{~mm}$. 


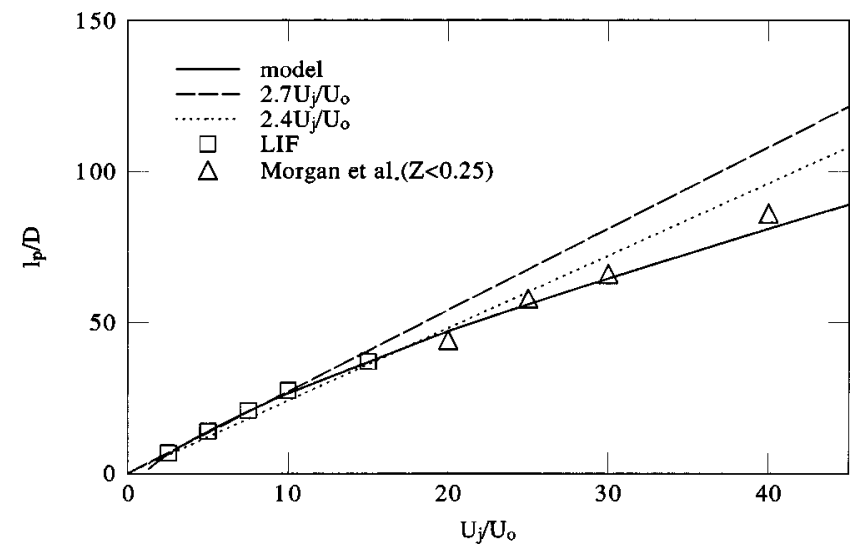

FIG. 8. Penetration distance at high jet-to-current velocity ratio:- present prediction; $\square$, LIF results from Lam and Chan (Ref. 5); $\triangle$, LIF results from Morgan et al.-Ref. $10(Z<0.25)$.

main counterflowing stream and the enclosing pipe to a remarkable degree. It was suggested that the flow is in the low jet momentum regime if the ratio $Z$ between the momentum flux of the jet and that of the main counterflowing stream is below 0.25 . Hence, we choose here to use their data of penetration distances in the low jet momentum regime at $U_{j} / U_{0}<40$ for their highest value of pipe-to-jet diameter ratio of 83. As shown in Fig. 8, their experimental values of $l_{p} / D$ at $U_{j} / U_{0}>15$ are also well predicted by our model. If we consider the momentum of the counterflowing stream in our laboratory flume, the momentum ratio $Z$ is below 0.15 even at our highest velocity ratio of 15 . Thus, our counterflowing jet experiments are well in the low jet momentum regime. Actually, our model is not expected to predict the jet penetration in the presence of significant confinement, the centerline velocity in a simple circular jet has been shown to drop in a manner other than the $1 / x$ decay law. ${ }^{1}$

\section{DISCUSSION}

We base our treatment of the centerline velocity decay of the axisymmetric jet in a counterflow on two physical processes. We assumed that fluid elements are discharged as if into a stagnant ambient and there is an advection effect from the counterflow on the fluid elements. The first fluid element leaving at the end of the potential core has a greater velocity gradient than in a stagnant ambient. It is primarily because its spatial width is reduced by the advection. With these two effects, the physical model proposed predicts very well the decay of mean centreline velocities.

We have not looked into the turbulence nature of the counterflowing jets and thus cannot discuss whether there are other flow interaction effects between the jet and the counterflow. However, the LIF results of Lam and Chan ${ }^{5}$ suggested that the spreading of the circular jet is enhanced by the counterflow and a linear instability analysis of Lam et $a{ }^{11}{ }^{11}$ suggested that the counterflow leads to selected amplification of jet shear-layer instabilities at lower frequencies and at the axisymmetric azimuthal mode. We can apply the simple assumption in this paper to explain these observations. Given that a section of the effluent jet fluid spreads in the same way as in the same jet after it leaves the jet exit, the countercurrent will compress the spatial coordinates so that when one observes successive fluid sections spreading while moving with the jet nozzle, one will see a larger spreading rate than in the simple jet. The instability of the counterflowing jet can similarly be explained by taking the effect of the counterflow as contracting the jet in the axial direction. Wavelengths of the most amplified modes are contracted so that the selected instabilities occur at lower frequencies, and higher order azimuthal modes are contracted to approximate the axisymmetric mode.

For a circular jet in a coflow, Michalke and Hermann ${ }^{12}$ have explored the idea of eliminating the external flow dependence of the jet flow by applying a stretching factor to the axial direction. A constant stretching factor was proposed and the resulting similarity was only approximate with respect to the instabilities which were taken to represent the large-scale turbulence structures of the jet. Our present treatment is consistent with the argument that the jet flow field is stretched or contracted in the axial direction by the external coflow or counterflow and that it may be possible to represent the effect of the external flow by a stretching or contracting of the axial coordinate. However, our treatment shows that it is not possible to apply a simple stretching factor of a constant value. Simple stretching or contracting occurs in the Lagrangian frame of reference on the fluid particles. When translating the flow into the Eulerian frame, the stretching effect becomes dependent on the local velocity and the integrated distance of travel.

\section{APPENDIX}

In our analysis, the velocity of fluid particle such as particle $\mathrm{A}$ is dropped by $\delta \nu$ as a result of momentum exchange with neighboring fluid elements. The situation is very similar to that described as follows. Consider a control volume with a width $d x$ at a position $x_{0}$ of a jet in a stagnant ambient. If we impose a velocity $-\delta \nu$ to it, the velocity gradient remains as $d U / d x=-K / x^{2}$ (where $K=U_{j} l_{M}$ ). The velocities at $x>x_{0}$ can be obtained by integrating the velocity gradient as

$$
\begin{aligned}
& \int_{K / x_{0}-\delta v}^{U} d U=\int_{x_{0}}^{x}-K / x^{2} d x, \\
& U(x)=K / x-\delta v .
\end{aligned}
$$

Thus, the velocity at the centerline will follow $K / x-\delta \nu$.

${ }^{1}$ N. Rajaratnam, Turbulent Jets (Elsevier, Amsterdam, 1976).

${ }^{2}$ H. W. Liepmann and J. Laufer, "Investigation of free turbulent mixing," N.A.C.A. Technical Note No. 1257, 1947.

${ }^{3}$ I. R. Wood, "Asymptotic solutions and behaviour of outfall flumes," J. Hydr. Eng., ASCE 119, 555 (1993).

${ }^{4}$ A. Andreopoulos and W. Rodi, "Experimental investigation of jets in a crossflow," J. Fluid Mech. 138, 93 (1984).

${ }^{5}$ K. M. Lam and H. C. Chan, "Investigation of turbulent jets issuing into a counterflowing stream using digital image processing," Exp. Fluids 17, 210 (1995).

${ }^{6}$ A. N. Sekundov, "The propagation of a turbulent jet in an opposing stream," Turbulent Jets of Air, Plasma and Real Gas, edited by G. N. Abramovich (Consultants Bureau, New York, 1969), pp 99-109. 
${ }^{7}$ M. Yoda and H. E. Fiedler, "The round jet in a uniform counterflow: Flow visualization and mean concentration measurements," Exp. Fluids 21, 427 (1996).

${ }^{8}$ H. B. Fischer, E. J. List, R. C. Y. Koh, J. Imberger, and N. H. Brooks, Mixing in Inland and Coastal Waters (Academic, New York, 1979).

${ }^{9}$ D. Liepmann and M. Gharib, "The role of streamwise vorticity in the near-field entrainment of round jets," J. Fluid Mech. 245, 643 (1992).

${ }^{10}$ W. D. Morgan, B. J. Brinkworth, and G. V. Evans, "Upstream penetration of an enclosed counterflowing jet," Ind. Eng. Chem. Fundam. 15, 125 (1971).

${ }^{11}$ K. M. Lam, S. K. Tang, and N. W. M. Ko, "The instability of a circular jet in a weak opposing stream," in Computational Mechanics. Proceedings of the Asian Pacific Conference on Computational Mechanics, Hong Kong, 1991, edited by Y. K. Cheung et al. (Balkema, Rotterdam, 1991), pp. $1435-1439$.

${ }^{12}$ A. Michalke and G. Hermann, "On the inviscid stability of a circular jet with external flow," J. Fluid Mech. 114, 343 (1982). 